\title{
CRYSTALLOGRAPHY
}

\section{Electron Density in Long Carbon-Carbon Bonds}

THE crystal structures of ammonium oxamate and lithium oxalate have been studied by $\mathrm{X}$-ray diffraction methods. Three-dimensional intensity data were collected with a xenon-filled proportional counter using copper $K \alpha$-radiation. Refinement of the three positional and six anisotropic vibrational parameters for each atom, including hydrogen, was carried out by the method of least squares; convergence occurred at $R=0.044$ for ammonium oxamate and $R=0.060$ for lithium oxalate. At this stage, three-dimensional $F_{o}-F_{c}$ Fourier syntheses were computed. These are shown in the form of superimposed sections in Figs. $1 a$ and $b$. Contours are drawn at intervals of $3 \sigma$, where $\sigma$ is the root mean square deviation of electron density averaged over the whole unit cell.

In each case an approximately spherical region of electron density, of height greater than $9 \sigma$, is found at the centre of the carbon-carbon bond; no other peaks higher than $4.5 \sigma$ or regions lower than minus $5 \sigma$ are present. Although in the case of lithium oxalate the peak lies on a centre of symmetry, the two determinations together provide strong evidence that a significant region of electron density exists at the centre of these carbon-carbon bonds.

The atomic scattering factors of Berghuis et $a l .^{1}$, which were used (modified for anisotropic vibrations) in the calculation of the structure factors, are apparently insufficient to describe exactly the electron density around the carbon atoms, although the valence state of the atom is considered. An exact fit of the electron density along the bond would be expected only if account were taken of the directional nature of the molecular orbital produced by the combination of the atomic orbitals.

In an attempt to remedy the discrepancy empirically, a correction was included, in the form of a fraction of a hydrogen atom placed at the mid-point of the carboncarbon bond. The fractions of McWeeny's scattering factor for hydrogen ${ }^{2}$ which were used were one-half for ammonium oxamate (an underestimate because it proved insufficient to account for the whole of the electron density peak) and two-thirds in the case of lithium oxalate. Vibrational

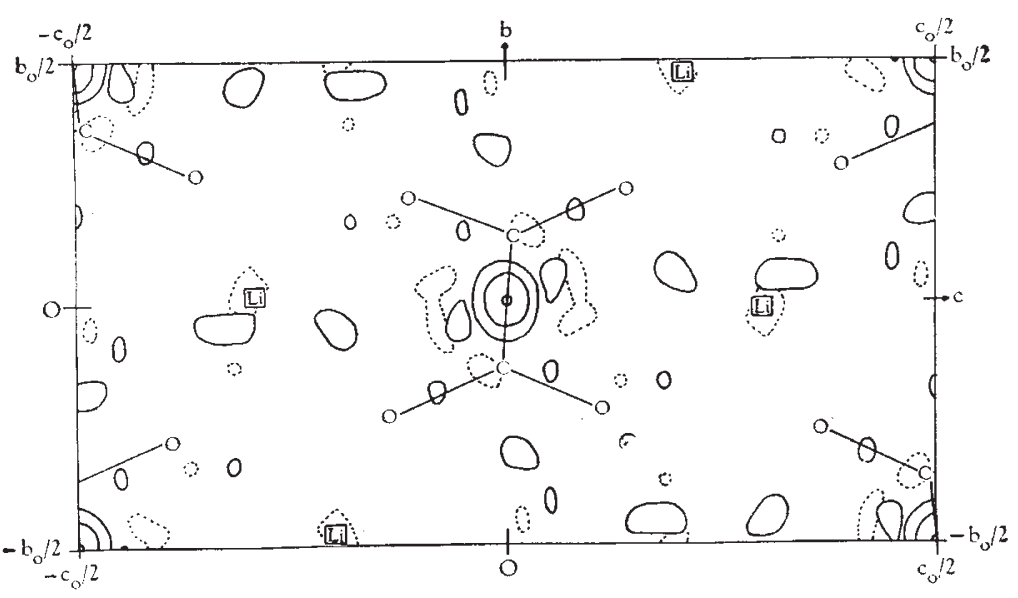

(b) Fig. 1. $F_{0}-F_{c}$ syntheses. $(a)$ ammonium oxamate; (b) lithium oxalate. Zero contours

ture factor data are deficient in terms of sufficiently short spacing to give effective resolution. Long bonds between atoms of comparatively low scattering power are therefore favourable situations in which to observe interatomic electrons.

B. Beagley

R. W. H. Small

Department of Chemistry,

University of Birmingham.

1 Berghuis, J., Haanappel, I. M., Potters, M., Loopstra, B. O., MacGillavry, (1955). ${ }^{2}$ McWeeny, R., Acta Cryst., 4, 513 (1951). ${ }^{3}$ Coulson, C. A., and Skancke, P. N., J. Chem. Soc., 2775 (1962). at the centre of the bond are as follows:

\section{Before allowance for electron density in bond}

After allowance for electron

$1.564 \pm 0.002$
Lithium oxalate

$1 \cdot 561 \pm 0.004$

$1.561 \pm 0.004$

Although the bond electron density correction did not affect the bond-length in the case of lithium oxalate, a change occurred in the orientation of the vibration ellipsoid. These results indicate, as would be expected, that the parameters of the neighbouring atoms tend to 'assimilate' the electron density in the bond during the least squares refinement.

The tendency of the atomic parameters to 'assimilate' interatomic electron density will be greater if the atoms are closer together, particularly if the experimental struc-

\section{Crystal Structure of Sinigrin}

SINIGRIN is a crystalline thioglucoside, extracted first by Bussy $^{1}$ from the seeds of black mustard. It is the potassium salt of the anion $\left[\mathrm{C}_{10} \mathrm{H}_{16} \mathrm{O}_{9} \mathrm{NS}_{2}\right]^{-}$, also called the myronate ion, and the crystals contain one molecule of water per potassium ion. In 1897 Gadamer $^{2}$ proposed formula (I) for the myronate ion. Schneider and collaborators confirmed the thioglycoside linkage in 1914 (ref. 3) and in 1930 (ref. 4) established that sinigrin and analogous glucosides are 1- $\beta$-D-thio-glucosides. Important details of the Gadamer formula I were challenged recently by 